\title{
Objective Investigation of Visual Function Using a Nondestructive Zoom-FFT Technique for Evoked Potential Analysis
}

\author{
M. P. Regan and D. Regan
}

\begin{abstract}
We describe an ultra-high resolution technique for recording evoked potentials (EPs) that are thousands of times smaller than the total EEG power. We report that two superimposed visual stimulus patterns, one modulated at $F_{1} \mathrm{~Hz}$ and the other at $F_{2} \mathrm{~Hz}$, can generate 20 or more EP frequency components, each of which is contained within a bandwidth of no more than $0.004 \mathrm{~Hz}$. We have developed a theoretical method for calculating the amplitudes and phases of these various cross-modulation components for different arrangements of model neurons. The relative amplitude of the various cross-modulation components seems to be a 'signature' of the particular neural model and this offers a way of testing theoretical neural models against EP data. As an illustration we compare the nonlinear EP components evoked by dichoptic stimulation with unpatterned flickering light against several models of binocular interaction. These nonlinear binocular interaction terms may offer a means of investigating binocularity in amblyopic children or infants who have low acuity in one or both eyes.
\end{abstract}

\begin{abstract}
RÉSUMÉ: Nous décrivons une technique à très haute résolution pour l'enregistrement du potentiels évoqués (PE) qui sont des milliers de fois plus petits que le total du potentiel EEG. Nous signalons que deux types de stimulus visuels superposés, l'un modulé à $F_{1} \mathrm{~Hz}$ et l'autre à $F_{2} \mathrm{~Hz}$, peuvent générer 20 composantes de fréquence des $\mathrm{PE}$ ou plus, chacune d'elle étant contenues à l'intérieur d'une bande de moins de $0.004 \mathrm{~Hz}$. Nous avons développé une méthode théorique pour calculer les amplitudes et les phases de ces différentes composantes de modulation croisée en tenant compte de différents arrangements de modèles neuronaux. L'amplitude des différentes composantes de modulation croisée semble être la "signature" d'un modèle neural particulier. Ceci permet d'éprouver des modèles neuraux théoriques en regard de l'enregistrement des PE. En guise d'illustration, nous comparons les composantes nonlinéaires des PE évoqués par stimulation dichoptique avec un faisceau lumineux scintillant nonmodulé en regard de plusieurs modèles d'interaction binoculaire. Ces termes d'interaction binoculaire nonlinéaires peuvent fournir un moyen d'investigation de la vision binoculaire chez les enfants amblyopes ou chez les nourissons qui ont une acuité visuelle réduite dans un oeil ou les deux yeux.
\end{abstract}

Can. J. Neurol. Sci. 1989; 16:168-179

Evoked potential (EP) recording is an objective means of investigating visual processing in the human brain, and in particular a means of isolating neural subunits in the visual pathway. For example, the technique has been used to study normal and abnormal functioning of the different mechanisms underlying colour vision, spatial form vision, binocular depth perception and motion perception (reviewed in 1,2).

The most widely-used recording procedures are as follows. (1) Stimulate the eye with an abrupt change along one visual dimension (e.g. an abrupt change of luminance or colour) and record the resulting EP by time-domain averaging. ${ }^{3}$ This is the familiar procedure used in recording clinical pattern VEPs ${ }^{4}$ and clinical auditory brainstem responses. ${ }^{5}$ (2) Stimulate the eye with a repetitive change along one visual dimension (e.g. a flickering light) and analyse the EEG into harmonics of the stimulus repetition frequency by sine/cosine multiplication. 1,2.6 Unfortunately, both these techniques have restricted specificity. For example, attempts to explore the orientation tuning of visual

From the Department of Psychology, York University (M.P.R.), and Department of Ophthalmology, University of Toronto (D.R.).

Received March 23, 1988. Accepted in final form October 25, 1988.

These data were presented in part at the Third International Evoked Potential Symposium, Berlin 1986.

Reprint requests to M.P. Regan, Department of Psychology BSB, York University, 4700 Keele St., North York, Ontario, Canada M3J IP3 
neurons by varying the orientation of a grating stimulus have been frustrated because the procedure does not select neurons tuned to a particular orientation, but rather evokes simultaneous responses from many sub-populations of neurons tuned to different orientations.

Greater specificity can be obtained by presenting two stimuli at the same time and analysing the cross-modulation components arising from nonlinear interactions between responses to the two stimuli. This approach can be understood as follows. If the input to a nonlinear system comprises the sum of two sinewaves, one of frequency $F_{1} \mathrm{~Hz}$ and the other of frequency $F_{2} \mathrm{~Hz}$, the output in general contains multiple discrete frequency components of frequencies $n F_{1} \pm m F_{2}$ where $n$ and $m$ are zero or integral. If $n=0$ the components are called 'harmonics of the stimulus frequency $F_{2}{ }^{\prime}$, and if $m=0$ the components are called 'harmonics of the stimulus frequency $F_{1}$ '. If neither $n$ or $m$ is zero, the components are called 'cross-modulation components'. The 'order' of any given cross-modulation component is equal to $(n+m)$. A linear system does not generate cross-modulation components. Our rationale is that a neuron can only generate cross-modulation products if it 'sees' both gratings at the same time. Thus, the cross-modulation products can be used to measure the orientation bandwidth of visual neurons. ${ }^{7}$ A similar problem arises in attempting to measure the spatial frequency tuning ${ }^{8.9}$ or temporal frequency tuning ${ }^{9}$ of visual neurons, and a similar solution has been described. The two-sinewave method has also been used to study short-range lateral interactions in the visual pathway. 10

Quite apart from estimating tuning bandwidths it has been suggested that the two-input technique might be used to identify the nature of the neural processing that gives rise to the nonlinear EP responses." A prerequisite here is to develop a theoretical method for calculating the responses of nonlinear neural models when stimulated by a two-sinewave input so that the predicted responses of different candidate models can be compared with EP data. Several authors have modelled individual neurons as asymmetric rectifier-like elements on the grounds that many neurons respond asymmetrically to, e.g. onset versus offset of light ${ }^{12}$ or leftward versus rightward motion. ${ }^{13}$ Previous theoretical work, however, has been limited to models containing a single rectifier stage. $10,11,14,15$ In view of the fact that the visual pathway comprises many sequential layers of neurons it seems likely that in some cases models involving several cascaded rectifier stages would be more satisfactory than singlerectifier models. With this in mind, we have developed a mathematical method for obtaining the responses of multiple-rectifier models to a two-sinewave input.2.16.17 Theoretical properties of cross-modulation terms for single rectifier models have previously been documented, but only for first and second-order (e.g. $F_{1} \pm F_{2}$ ) terms, ${ }^{14,15,18}$ and the theoretical properties of crossmodulation terms for multiple-rectifier models have not been previously documented at all. Here and elsewhere 2.17 we describe the theoretical properties of terms up to the fifth order (i.e. $n+m=5$ ).
In order to compare neural models with EP data it is necessary to measure many simultaneously-occurring frequency terms in the EEG, some of which have very low amplitudes. We describe here an ultra-high resolution method for recording the steady-state EP that allows precise measurement of components whose power is several thousand times less than the overall EEG power. By way of illustration, these recording and theoretical methods are applied to investigating the binocular interaction between monocular responses to flickering light.

\section{MethodS}

\section{Zoom-FFT and its Application to Evoked Potential Recording}

Repetitive sensory stimulation generates a steady-state EP consisting of several discrete frequency components. These components can be extracted from the EEG by analog sine/cosine multiplication 6 or by a digital computer technique called the Fast Fourier Transform (FFT). The FFT produces a plot of EEG power versus frequency, called a power spectrum (or alternatively a plot of voltage versus frequency, called an amplitude spectrum) covering a range from DC to some chosen maximum frequency $\mathrm{f}_{\text {max. }}$. The best possible frequency resolution of this spectrum is set by the duration of the EEG time signal: the longer the duration, the better the resolution. In particular, the best possible frequency resolution $(\Delta F)$ for an EEG sample of duration $\mathrm{T} \mathrm{sec}$ is given by $\Delta \mathrm{F}=1 / T \mathrm{~Hz}$. (This equation exists in several slightly different versions, depending on how "frequency resolution" is defined). ${ }^{19}$ Thus, for a DC- $100 \mathrm{~Hz}$ bandwidth recording of $250 \mathrm{sec}$ duration, this theoretical limit is about $0.004 \mathrm{~Hz}$, giving about 25,000 lines over DC- $100 \mathrm{~Hz}$ bandwidth. However, there is often a practical problem in achieving this best possible resolution or even approaching it. Suppose that the EEG recording bandwidth is chosen to be DC$100 \mathrm{~Hz}$. A digital analyser samples the EEG waveform at some preset frequency, and this sampling frequency must be set at rather more than twice the highest frequency in the EEG signal. Suppose that the sampling frequency is $250 \mathrm{~Hz}$ and the recording duration is 256 seconds, then the total number of samples will be $250 \times 256=64,000$. An FFT carried out on these 64,000 samples as a whole would give a spectrum with a resolution of $(256)^{-1} \mathrm{sec}$, that is approximately $0.004 \mathrm{sec}$, i.e. the theoretical resolution for a $256 \mathrm{sec}$ recording duration. Using present technology, however, a computer with sufficient memory to store such a large number of samples, each of which is measured with sufficiently high precision, would be very expensive indeed, and the computations required to obtain the spectrum might be time-consuming. Zoom-FFT is a procedure for transcending this technical limitation so as to generate a spectrum with the best possible resolution $\Delta F=1 / T$ on the basis of a comparatively small number of time samples. ${ }^{20}$ It follows that only a correspondingly small part of the original frequency range can be covered in any one analysis. 
Fast, special-purpose FFT analyzers are available but they operate with a rather low number of time samples. Rather than the $\mathrm{N}=64,000$ time samples we have just discussed FFT analyzers are commonly restricted to $\mathrm{N}=1024$ or $\mathrm{N}=2048$ samples. The FFT transforms the $\mathrm{N}$ equally-spaced time samples of the EEG into $N / 2$ equally-spaced lines in the frequency domain, so that the resolution over a DC- $f_{\max }$ bandwidth is about $2 f_{\operatorname{mar}} / N$ independently of the recording duration. (For technical reasons, however, the situation is even worse: rather less than $\mathrm{N} / 2$ lines are usually available.) The following paragraph explains how zoom-FFT can be achieved by frequency shift (heterodyning) followed by low-pass filtration.

Suppose we wish to concentrate our entire number of available lines (e.g. 800) within the frequency range $f_{k}$ to $\left(f_{k}+3.125\right)$ rather than over the entire DC- $100 \mathrm{~Hz}$ bandwidth. We must deal with the problem that the FFT gives a spectrum with a lowest frequency of zero (i.e. DC) rather than $f_{k}$. First, the EEG signal is lowpass filtered by the anti-aliasing filter before being sampled by the analog to digital converter (ADC). This ensures that the highest frequency in the signal is less than half the sampling rate $f_{s}$. For our DC- $100 \mathrm{~Hz}$ EEG, let us take $f_{s}=250 \mathrm{~Hz}$. The samples from the ADC are then multiplied by a complex exponential function $e^{-i 2 \pi f_{k} l}$, i.e. by a unit vector rotating at a rate $-f_{k}$. This multiplication produces the desired frequency shift, moving the frequency span of interest from $f_{k}$ to $\left(f_{k}+3.125\right)$ to (DC$3.125) \mathrm{Hz}$. After this frequency shift the time samples are fed through a low-pass filter that passes only the frequency span of interest (i.e. DC-3.125 Hz) and resampled at a rate $\left(f_{s} / z\right)$ where $z$ is the zoom ratio. (In this case $z=100 / 3.125=32$.) Finally, the time samples are subjected to FFT.

Unfortunately, the original time function is destroyed by the zoom operation, so that only a limited section of the entire frequency spectrum can be analyzed. We have found that high zoom ratios are needed to exploit the high signal-to-noise ratios consequent on the very narrow bandwidths of the steady-state EP's discrete frequency components. For example with an 800line machine we require a zoom ratio of about 32 to give the $0.0039 \mathrm{~Hz}$ bandwidth of Figure 1. But also we typically require to quantify 30 or more different discrete frequency components that can be located virtually anywhere within our DC-100 Hz bandwidth.

The time factor cannot be ignored either. For example, in practice to analyze any given $3.125 \mathrm{~Hz}$ segment of the DC-100 bandwidth into 800 discrete frequency bins at a resolution of $0.0039 \mathrm{~Hz}$ calls for a recording duration of at least 256 seconds. But if the analysis of each and every $3.125 \mathrm{~Hz}$ segment of the DC- $100 \mathrm{~Hz}$ bandwidth took 256 seconds the zoom-FFT analysis of one 256 second recording would take over 2 hours!

Our solution to this problem is to use a specially-developed procedure that allows high zoom ratios to be combined with zoom-FFT analysis that is both nondestructive and fast.

In brief, the real-time analog EEG signal is fed to Bruel and Kjaer model 2032 analyzer that digitizes it at a rate appropriate to the EEG bandwidth (e.g. $250 \mathrm{~Hz}$ for a $100 \mathrm{~Hz}$ bandwidth), and places the digitized time signal in a temporary store. Then the digitized time signal is played at a much increased speed into a Hewlett-Packard model 9000 computer where it is permanently stored on floppy disk. Off-line analysis is achieved under the control of the model 9000 computer at a much higher rate than the original digitizing rate. Thus, for example a segment of EEG that took $320 \mathrm{sec}$ to record is replayed in less than $2 \mathrm{sec}$. The analyzer then carries out a destructive zoom-FFT at the desired zoom ratio (e.g. 32-fold).

Included in our model 9000 software is an optional automatic analysis. Before starting the experiment the stimulus frequency or frequencies are fed into the analyzer. The model 9000 computes the values of $F_{1}, F_{2}, 2 F_{1}, 2 F_{2}, F_{1}+F_{2}, 2 F_{1}+2 F_{2}$ etc.

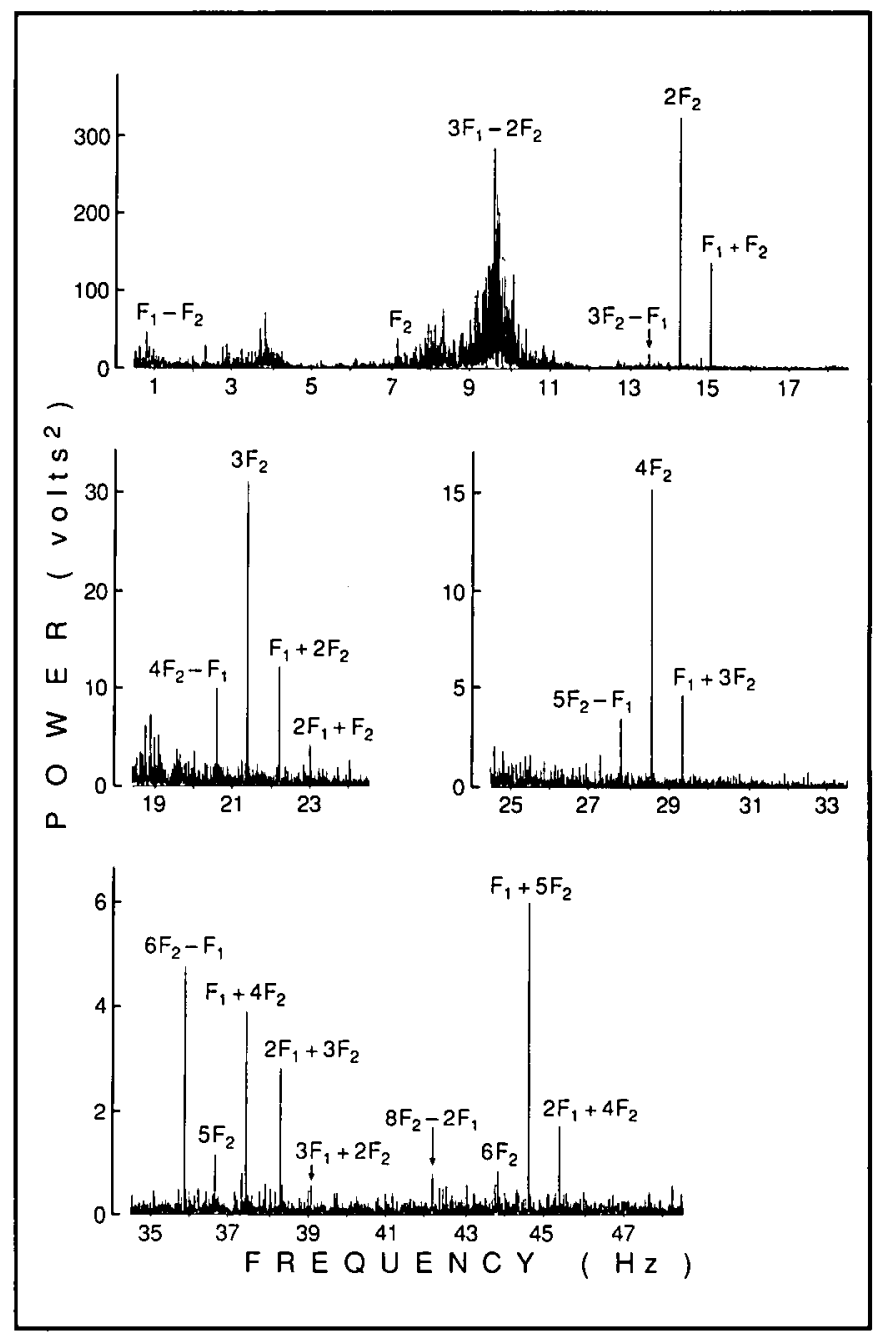

Figure $1-A$ homogeneous circular white patch of light of diameter 10 deg and mean luminance $250 \mathrm{cdm}^{-2}$ was multiplied by the sum of two sinusoids, one of frequency $8 \mathrm{~Hz}$, giving a modulation depth of $20 \%$, and one of frequency $7 \mathrm{~Hz}$, giving a modulation depth of $60 \%$. The EEG was analyzed by nondestructive zoom-FFT at a resolution of $0.0039 \mathrm{~Hz}$. The zoom ratio was 32 . Recording duration was 320 sec. This section contains 12,300 lines over $0.5-49.5 \mathrm{~Hz}$ bandwidth. The steady-state EP consists of discrete frequency components whose bandwidths were less than $0.0039 \mathrm{~Hz}$. Cross modulation terms are due to nonlinear interactions between neural responses to the two flickers. 
up to a specified number of terms and with a precision of 7 significant figures. These values are stored by the model 9000 computer and used to identify response components. Immediately after the model 2032 analyzer has completed a zoom-FFT analysis, our model 9000 computer software searches for signals at the pre-specified frequencies. Unless a measured 'spike' agrees with one of the predicted frequencies to within one bin, the spike is not accepted as a signal. The computer measures the mean power in the central 'spike' and in the two immediately-adjacent bins. It also estimates the noise level in that local frequency region by taking into account the power in the neighbouring (e.g. 10) bins on either side of the signal (see below for discussion on quantifying signal-to-noise ratios).

Successive segments of the DC-100 Hz signal are analyzed by repetitions of this procedure. Although each successive zoom-FFT destroys the EEG signal in the model 2032 analyzer it is replaced every time by rapid transfer from the EEG time signal permanently stored within the model 9000 computer. Permanent storage of the original time signal also allows further analysis months or years after the data are collected.

Figure 1 illustrates part of a DC- $100 \mathrm{~Hz}$ EEG signal analyzed by this procedure at a resolution of $0.0039 \mathrm{~Hz}$. There are about 12,500 frequency bins between 0.5 and $49.5 \mathrm{~Hz}$ in Figure I, i.e. 12,500 lines. Note that the ordinate in the $34.5-48.5 \mathrm{~Hz}$ segment is 50 times smaller than in the $0.5-18.5 \mathrm{~Hz}$ segment and that the $5 F_{2}$ component is quite clear, even though it is about 300 times smaller than the $2 F_{2}$ component and its power is thousands of times less than the total EEG power.

The extreme narrowness that a steady-state evoked potential component can attain is illustrated in Figure 2. The subject viewed a counterphase-modulated grating for 640 secs, thus giving the analyzer a resolution of $0.00195 \mathrm{~Hz}$. Figure 2 shows

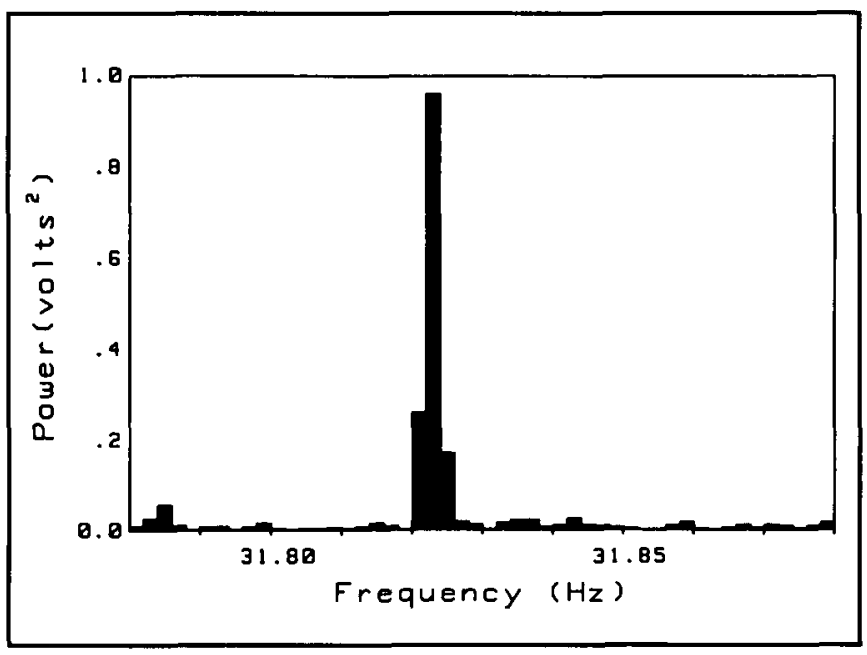

Figure 2 - A single vertical grating of contrast $40 \%$ was counterphase modulated at $F=8 \mathrm{~Hz}$, and viewed binocularly. The EEG was recorded for 640 secs, allowing the analyser to attain a frequency resolution of $0.00195 \mathrm{~Hz}$. The zoom ratio was 64. The plot shows the relative power per bin at and around the $4 \mathrm{~F} \mathrm{~Hz}$ evoked potential component, demonstrating the remarkable narrowness of the response component. that the experimentally-measured EP power was mostly contained in one $0.00195 \mathrm{~Hz}$ bin. Thus, the bandwidth of the EP component was no more than $0.00195 \mathrm{~Hz}$, and perhaps even less. (Even if the signal had had indefinitely narrow bandwidth, some power would have spread into adjacent lines because of overlap between the sensitivity curves of adjacent bins. In particular, the total power in the two adjacent lines cannot be less than about $50 \%$ of the power in the central line, even for a signal of indefinitely narrow bandwidth - and this is approximately what we see in Figure 2). This narrowness in the frequency domain implies that the amplitude and phase variability of the signal was small. ${ }^{2}$

The zoom-FFT technique provides an overview of the frequency components that are present. When one already knows which frequency components are of interest, a second technique is possible. A simple analog Fourier Series analyzer 1.2 .6 will extract any given term of $\left(n F_{1} \pm m F_{2}\right)$ when provided with a reference sine and cosine of the appropriate frequency. This technique provides phase as well as amplitude, and can easily be incorporated with the rapid 'EP sweep' technique.2.21,22 A simple method for generating a reference sine and cosine of frequency $\left(n F_{1} \pm m F_{2}\right)$ is available (Ref. 2, Section 1.8.6).

Finally, we should note some of the reasons why it is necessary to use the unfamiliar technique described above rather than the familiar averaging computer when recording responses such as the EP of Figure 1. The chief reason is that most of the EP terms in Figure $I$ are not related harmonically, i.e. they are not an integral multiple of some 'first harmonic' or 'fundamental' frequency. Consequently, a conventional time-domain averager would reject many of the EP components in Figure I because a conventional averager requires a trigger, and acts as a comb filter that rejects all frequencies except harmonics of the trigger frequency. ${ }^{3}$ These rejected components are destroyed irrecovably, and cannot be recovered by subjecting the time-averaged EP to frequency-domain analysis e.g. by the FFT. (This point is illustrated in Fig.1.32 in Ref. 2). In particular: Fourier transform followed by frequency-domain averaging can produce a quite different result than time-domain averaging followed by Fourier transform. In Figure I, each of $\mathrm{N}$ samples of EEG was subjected to spectral analysis, and only then did averaging take place (the $\mathrm{N}$ spectra were averaged). Figure 1 illustrates that this procedure preserves non-harmonic as well as harmonic components of the steady-state EP. Note the crucial point that, in contrast with the familiar time-domain averaging procedure, frequency-domain averaging does not need a trigger and that consequently an indefinitely large number of non-harmonic components can be extracted in a single spectrum average.

\section{Signal-to- Noise Ratio : the Tradeoff Between More Averages and Narrower Analysis Bandwidth}

Familiarity with the time domain averaging implemented by the conventional 'averager' can leave one with the impression that an EP is buried in noise when its amplitude is less than the amplitude of the total residual EEG 'noise' in the final recording, and that an EP is reasonably clear when its amplitude is 
about 2-3 times the amplitude of the total 'noise'. This is indeed true for time domain averaging of transient EPs. But a moment's reflection shows that it is not true at all for frequency-domain analysis of steady-state EPs, where an EP whose power is thousands of times smaller than the total residual EEG power in the final recording can often be quite clearly seen (e.g. the $5 F_{2}$ component in Figure 1). For steady-state EPs analyzed in the frequency domain, the clarity of a signal is determined by properties of the noise in the immediate neighbourhood of the signal frequency rather than simply by the overall amplitude of the noise as is the case in time-domain averaging. 1,6

In Figure 3A we suppose that there is equal noise power in every bin near the $15.000 \mathrm{~Hz}$ signal. Consequently, the signal
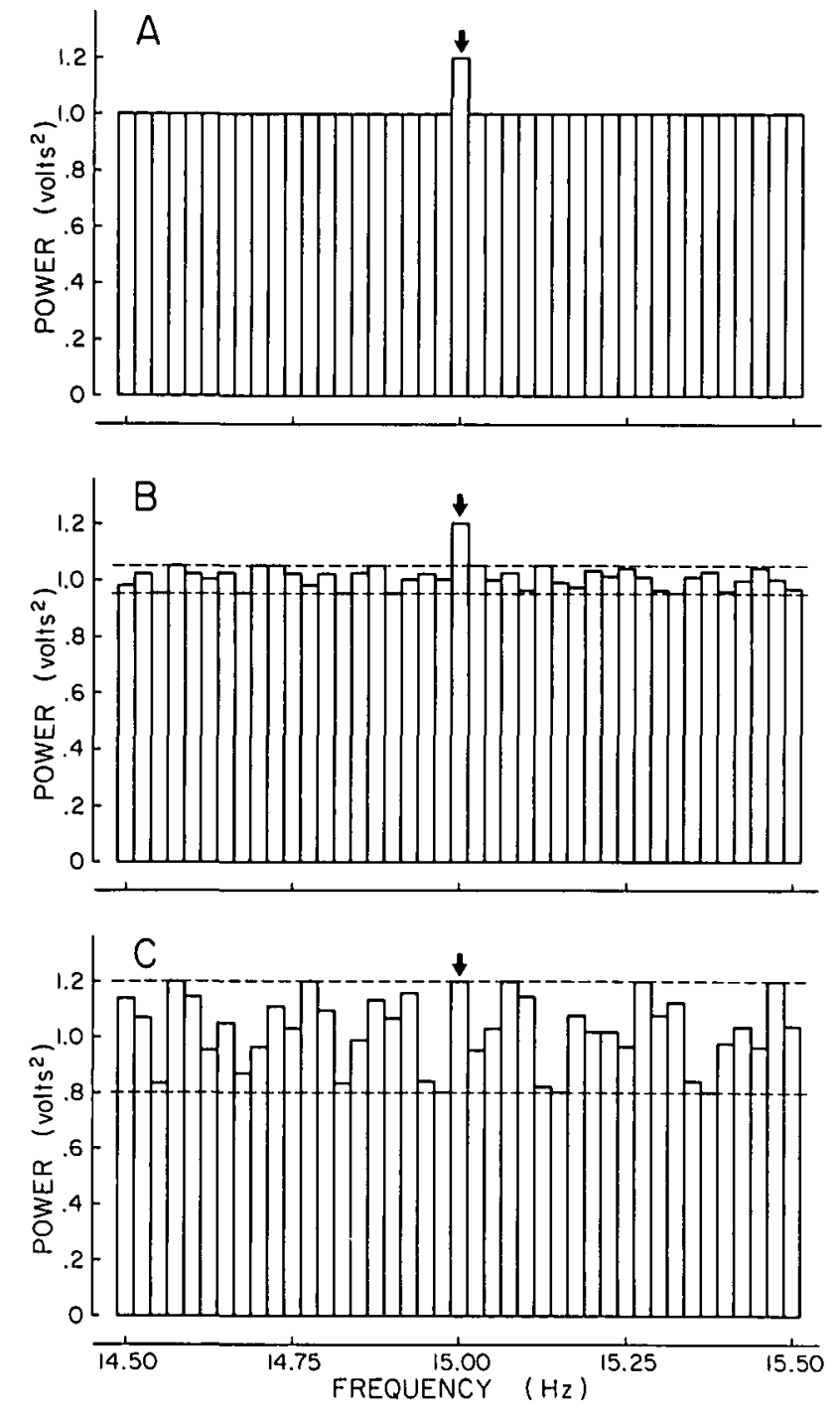

Figure 3 - The signal-to-noise ratio of a spectral component depends on the variability rather than the level of power in adjacent bins. The mean noise power in adjacent bins is the same (1.0) in $A$ through $C$, but the same signal (arrowed) is clear in $A$ and buried in $C$. (arrowed) is clearly visible even though its power is only $20 \%$ greater than the noise power in any adjacent bin. In effect, its signal-to-noise ratio is infinitely large. This reducto ad absurdem illustrates that it is not the absolute power of noise in adjacent bins that determines signal-to-noise ratio, but rather the bin-to-bin variability of signal power in adjacent bins. This point is brought out in Figures $3 \mathrm{~B}$ and $\mathrm{C}$. Although the mean noise power in adjacent bins is the same (1.0) in Figures $3 \mathrm{~A}, \mathrm{~B}$ and $\mathrm{C}$, the bin-to-bin variations increase to $\pm 5 \%$ peak-to-peak in Figure 3B and $\pm 20 \%$ peak-to-peak in Figure 3C, at which point the $15.000 \mathrm{~Hz}$ signal is entirely buried in noise.

We can now understand that the experimenter who wishes to improve signal-to-noise ratio should focus on increasing the power of the signal 'spike' relative to the variability of power in adjacent bins. Next we discuss how this aim can best be achieved.

The bandwidth of an infinitely long sinewave is infinitely narrow so that, as the recording duration is made longer and longer, the bandwidth of the recorded signal grows narrower and narrower. In practice, however, the bandwidth of a real steady-state EP component cannot be made indefinitely narrow by recording the EEG for an indefinitely long time. This is because the amplitude and phase of an EP signal vary from
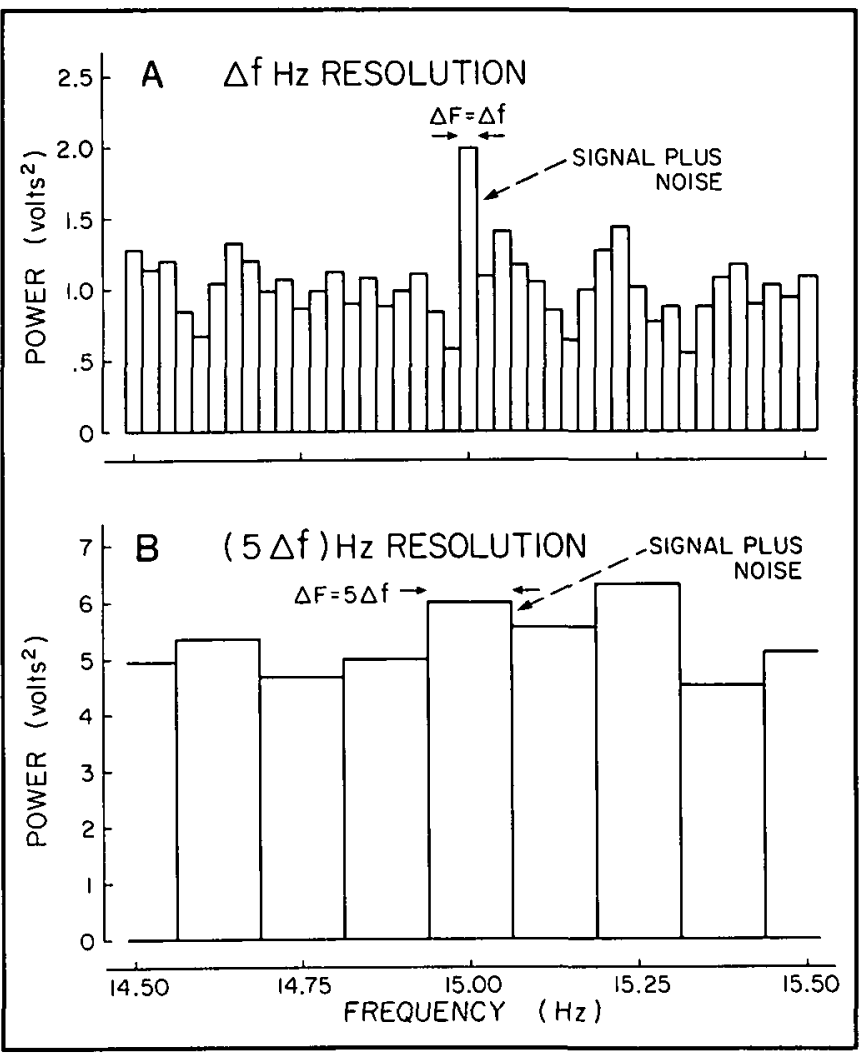

Figure 4 - Matching the spectrum analyzer's bandwidth to the signal bandwidth gives optimal signal-to-noise ratio. If the recording duration and number of bins are selected so that the signal bandwidth $\Delta f$ is matched by the analyzer's bandwidth $\Delta F$, the signal is clear in this example (Panel $A$ ), but if the analyzer's bandwidth is widened the signal is lost (Panel B). 


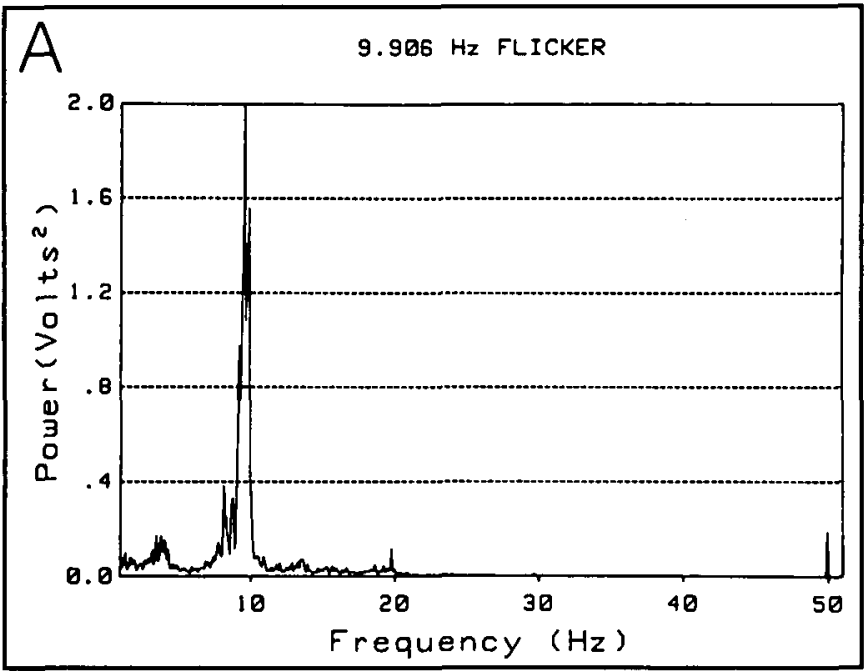

A

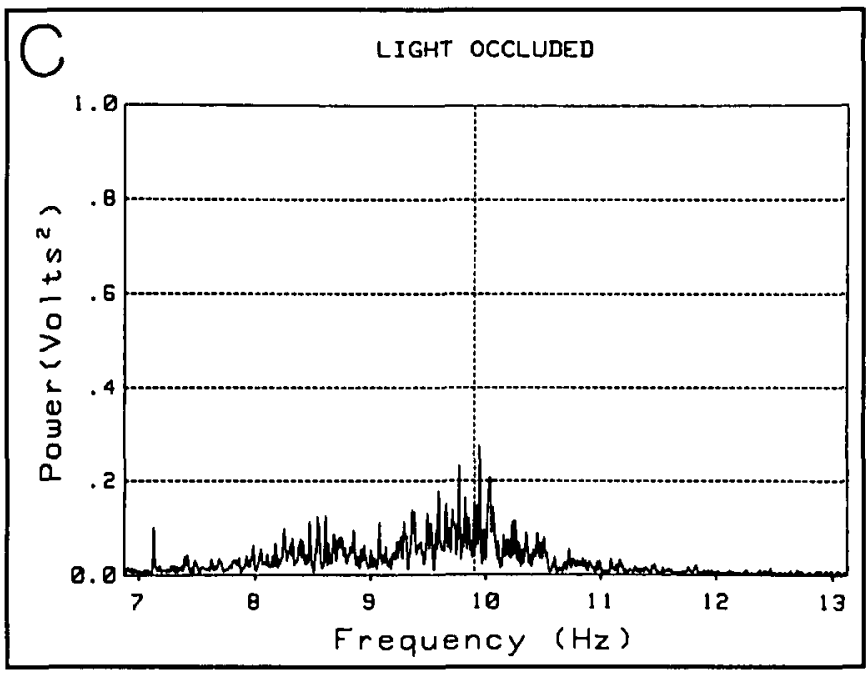

C

moment to moment, and the effect of these variations is to create sidebands and to broaden the bandwidth of the EP signal. The bandwidth of our $15.000 \mathrm{~Hz}$ EP component is shown as $\Delta f$ $\mathrm{Hz}$ in Figure 4. As mentioned above, the frequency resolution $(\Delta F \mathrm{~Hz})$ of a spectral analyzer cannot be less than $(\Delta T)^{-1}$ where $\Delta T$ is the recording duration. Suppose we choose a recording duration such that the resolution of the analyzer is equal to the bandwidth of the EP component, i.e. we record for a duration $\Delta T \sec$ where $(\Delta T)^{-1}=\Delta F=\Delta f$.

For the purpose of the following simplified account we assume : [1] that the signal in Figure 4A falls into exactly one bin that contains a total power of 2.0 arbitrary units, [2] that the mean 'noise' power in adjacent bins is 1.0 , and [3] that the binto-bin variation is normally distributed with a standard deviation of $20 \%$. Suppose, for sake of argument that the noise power in the $15.000 \mathrm{~Hz}$ bin is exactly 1.0 and this adds with the signal to give the 2.0 ordinate. Figure $4 \mathrm{~A}$ shows that the signal is reasonably clear.

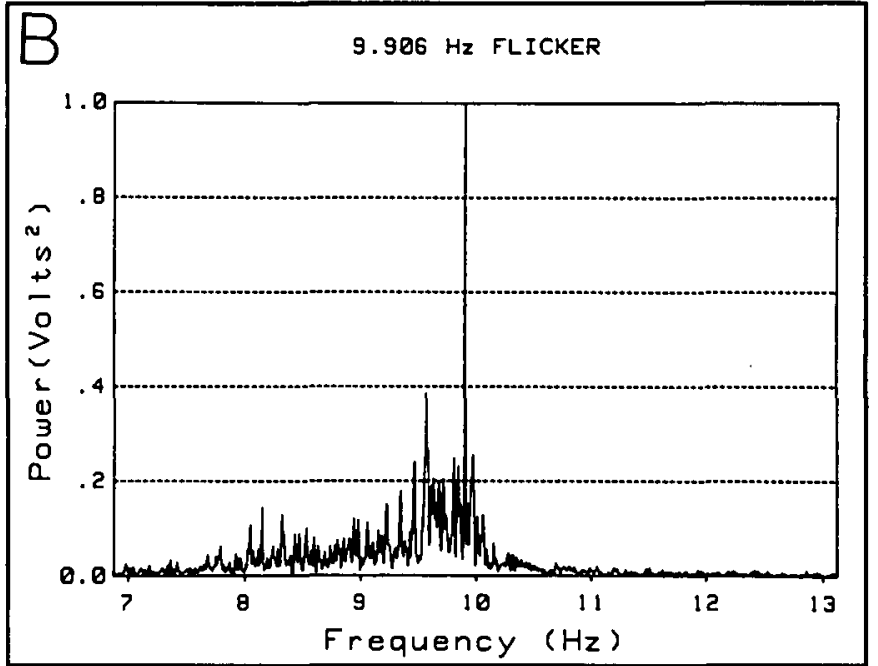

$\mathbf{B}$

Figure $5 A$ - The EEG was analysed at a frequency resolution of 0.0625 $\mathrm{Hz}$ while the subject viewed a light whose intensity was sinusoidally modulated at $9.906 \mathrm{~Hz}$.

Figure $5 B$ - The same EEG sampled analyzed at a frequency resolution of $0.00781 \mathrm{~Hz}$. A $9.906 \mathrm{~Hz} E P$ is clearly visible in panel B hut not in panel $A$

Figure $5 C$ - As in panel B except that the light was occluded by black card. The dotted line is a marker at $9.906 \mathrm{~Hz}$. Recording duration 320 sec.

Suppose now that the analyzer's bandwidth is made five times coarser than the signal bandwidth, i.e. $\Delta F=5 \Delta f$ (Figure 4B). The $15.000 \mathrm{~Hz}$ bin will now have total power 6.0 because it contains the 1.0 signal power previously contained in the $15.000 \mathrm{~Hz}$ bin in Figure 4A plus the noise previously contained in the adjacent five bins in Figure 4A. Adjacent bins in Figure $4 \mathrm{~B}$ will contain an average power of 5.0 because they are 5 times wider than the bins in Figure 4A. Bin-to-bin variability will be reduced by a factor of about $\sqrt{5}$ but this will not compensate for the reduction in the prominence of the 'signal bin' from 2:1 to 6:5 with respect to the mean height of adjacent bins.

Summarizing, the signal-to-noise ratio is improved by reducing the bandwidth $(\Delta F \mathrm{~Hz})$ of the analyzer until it matches the bandwidth $\Delta f \mathrm{~Hz}$ of the signal, and this requires that an appropriately long duration ( $\Delta T \mathrm{sec}$ ) of EEG is analyzed [i.e. $\Delta T \geq(\Delta F)^{-1}$ ]. Figure 5 is a practical illustration of this point. A stimulus flicker frequency of $9.906 \mathrm{~Hz}$ was chosen so that the fundamental component of the steady-state EP would fall in the 
high-noise alpha region, so as to present the analyser with a difficult task of signal-to-noise enhancement. In panel $\mathrm{A}$ the analyzer's resolution is $0.0625 \mathrm{~Hz}$ and this is evidently inadequate. There are two spikes near $9 \mathrm{~Hz}$, and it is not clear whether either of them is a signal. Panel B presents a quite different picture. This shows the same EEG data analyzed at eightfold higher resolution (i.e. $0.0078 \mathrm{~Hz}$ ). The $9.906 \mathrm{EP}$ component is clearly seen. The reason why the EP component is clear in panel B but not in $\mathrm{A}$ is that its bandwidth is evidently no more than $0.0078 \mathrm{~Hz}$ so that the eightfold increase of analyser resolution capitalizes on the signal's very high concentration of power per $\mathrm{Hz}$ : almost all the signal power is restricted to one bin in both $B$ and $A$, but each bin in $B$ contains on average 8 times less noise power than in $A$ because the frequency resolution is eight times more precise. Panel $\mathrm{C}$ is a "light occluded" control to demonstrate that the spike in B is not an artifact.

The policy of sharpening the analyzer's frequency resolution by increasing the length of EEG analyzed can, however, be pushed too far. The EP is generated by a human subject who is prone to fatigue and boredom so that, if the recording duration is too long, the moment-to-moment variability will increase and hence the bandwidth of the EP component will broaden. Thereafter, as the analyzer's bandwidth is progressively falling, so the bandwidth of the physiological EP signal is widening. The experimenter must use trial and error to estimate the recording duration that gives the best compromise.

Our efforts so far have been directed to increasing the size of the signal 'spike' relative to the variability of adjacent bins. An alternative approach is to reduce the absolute variability of adjacent bins. This can be achieved by averaging in the frequency domain. It may be that moment-to-moment variations of EP amplitude and phase remain constant over a prolonged period. In which case it is advantageous to record a longer section of EEG than is required to match the bandwidth of the analyzer to the bandwidth of the EP signal. For example, suppose we have recorded a continuous 256-sec sample of EEG. In the extreme cases we may select a $0.0125 \mathrm{~Hz}$ resolution that requires an 80 sec recording time and reduce bin-to-bin variability by frequency-domain averaging, or we may use the whole 256-sec sample to achieve a frequency resolution of $0.0039 \mathrm{~Hz}$. Which of these choices will give a better signal-to-noise ratio depends on the properties of the EP signal. If the EP bandwidth over a 256-sec recording duration is substantially greater than $0.0039 \mathrm{~Hz}$ it is best to use a lower analyzer bandwidth with some averaging.

Note that, even if the EP signal approximated a sinewave of constant amplitude and phase over the first minute of recording time, one could not in principle fully capitalize on this fact if the moment-to-moment EP variability increased substantially over the succeeding minutes, because no analyzer can achieve a very narrow bandwidth within a short recording duration: $\Delta F \geq(\Delta T)^{-1}$ is inescapable. ${ }^{19}$ The optimal trade-off between analyzer bandwidth and number of frequency-domain averages depends on the way in which the particular EP signal's momentto-moment variability changes with time and the way in which adjacent bin-to-bin variability changes with time. This can only be determined by trial and error.

\section{Stimulation}

The stimulus consisted of two homogeneous circular white fields subtending $10 \mathrm{deg}$, of equal luminances, viewed at 100 $\mathrm{cm}$. The luminance of one field was modulated sinusoidally to a depth of $20 \%$, at $F_{1}=8 \mathrm{~Hz}$ and the other was modulated at $F_{2}=7 \mathrm{~Hz}$ and the modulation depth varied from $0 \%$ to $80 \%$. The two fields were presented dichoptically. Great care was taken to ensure that $F_{1}$ flicker was routed entirely to one eye and $F_{2}$ flicker entirely to the other. The two stimuli were led to the eyes by light-tight tubes connected to light-excluding goggles. The goggles contained a black foam septum to maintain eye segregation. The absence of optical cross-talk was confirmed by checking that when one eye's stimulator was switched off that eye saw no flicker, even when the fellow eye's stimulator was $100 \%$ modulated.

\section{RESULTS}

\section{Theoretical}

Using a double Fourier integral method based on a mathematical approach suggested by Bennett ${ }^{18}$, one of us (MPR) has developed a theoretical method for obtaining the response of multiple-rectifier models to an input comprising the sum of two sinewaves of frequencies $F_{1}$ and $F_{2} \mathrm{~Hz}^{2,17}$ As already mentioned, the output contains multiple discrete frequencies $\left(n F_{1} \pm m F_{2}\right) \mathrm{Hz}$. For example, Figure 6A shows how the amplitudes of these response components vary with $k$ for the cases of a single half-wave square-root rectifier. ( $k$ is the ratio of the amplitudes of the $F_{1} \mathrm{~Hz}$ and $F_{2} \mathrm{~Hz}$ inputs). Components up to the fifth order $(n+m=5)$ only are shown, but higher-order terms are readily calculated.

Figure $6 \mathrm{~A}$ brings out a point that is not intuitively obvious, but in practical recording is of first importance. Some response components are only appreciable at specific values of $k$ - see, for example, the $\left(2 F_{1}+2 F_{2}\right),\left(4 F_{1}+F_{2}\right)$ and $\left(3 F_{1}+2 F_{2}\right)$ terms. Thus, if the stimulus were an $F_{1} \mathrm{~Hz}$ flickering light of $20 \%$ modulation depth superimposed on an $F_{2} \mathrm{~Hz}$ flickering light of variable modulation depth the $\left(4 F_{1}+F_{2}\right)$ EP term would be seen when the $F_{2}$ modulation depth was near $8 \%$, but would be weak or absent when the $F_{2}$ modulation depth was reduced below about $4 \%$ or increased above about $15 \%$.

We have obtained families of curves of the type shown in Figure 6A not only for single rectifiers (compressive, linear accelerating, and more complex shapes including one that resembles the contrast transducer function of the human eye), but also for multiple cascaded rectifiers and the parallel-cascaded case ${ }^{17}$ (i.e. the dichoptic case, Figure 6B).

Figure 7 illustrates the point that different rectifier models can produce families of curves that look rather similar when only low-order terms are considered, but become more and more distinctive as higher and higher order terms are taken into consideration. The four rectifier models considered are: (1) A linear rectifier followed by a compressive (thirdroot) rectifier, 


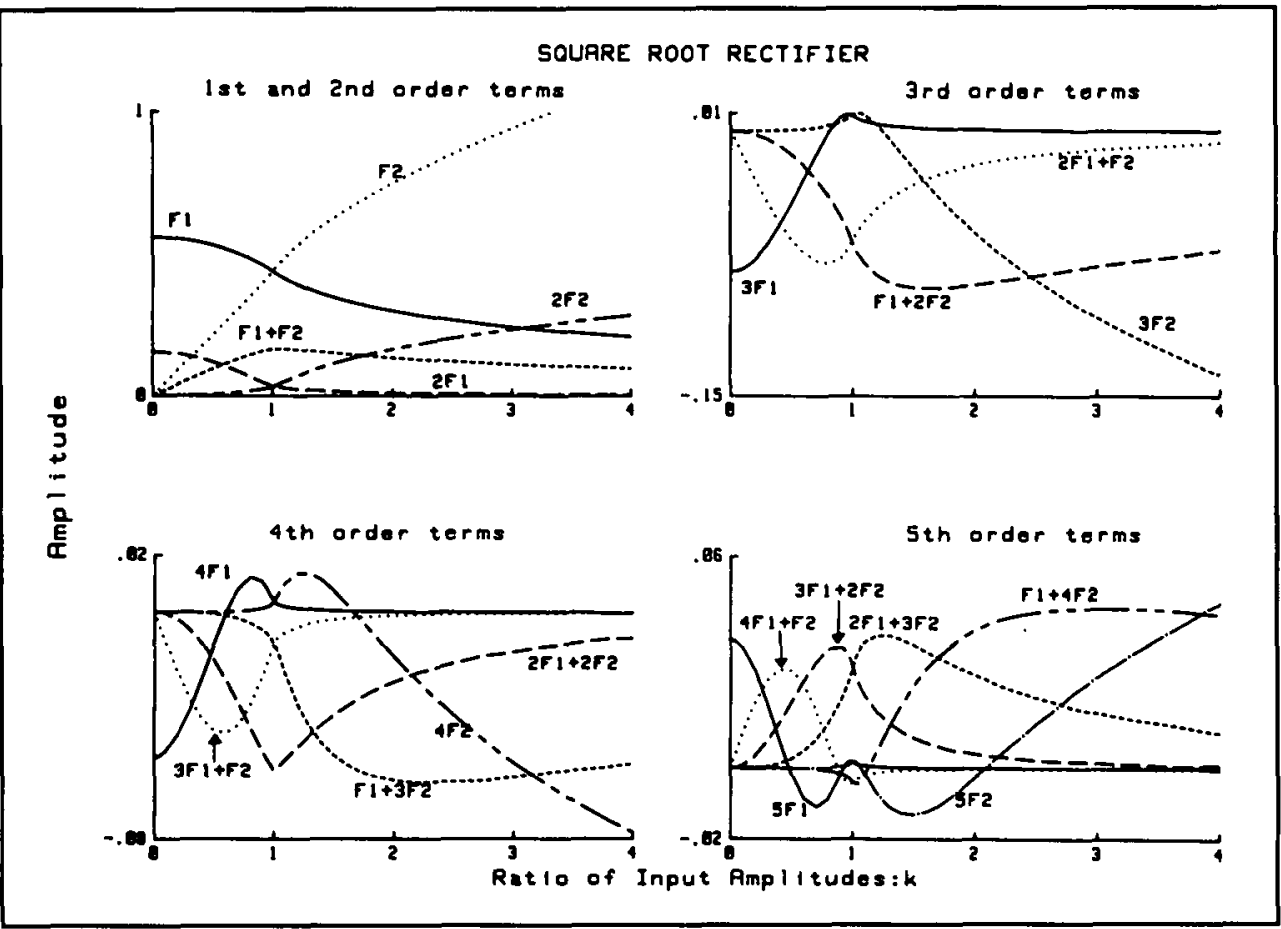

A

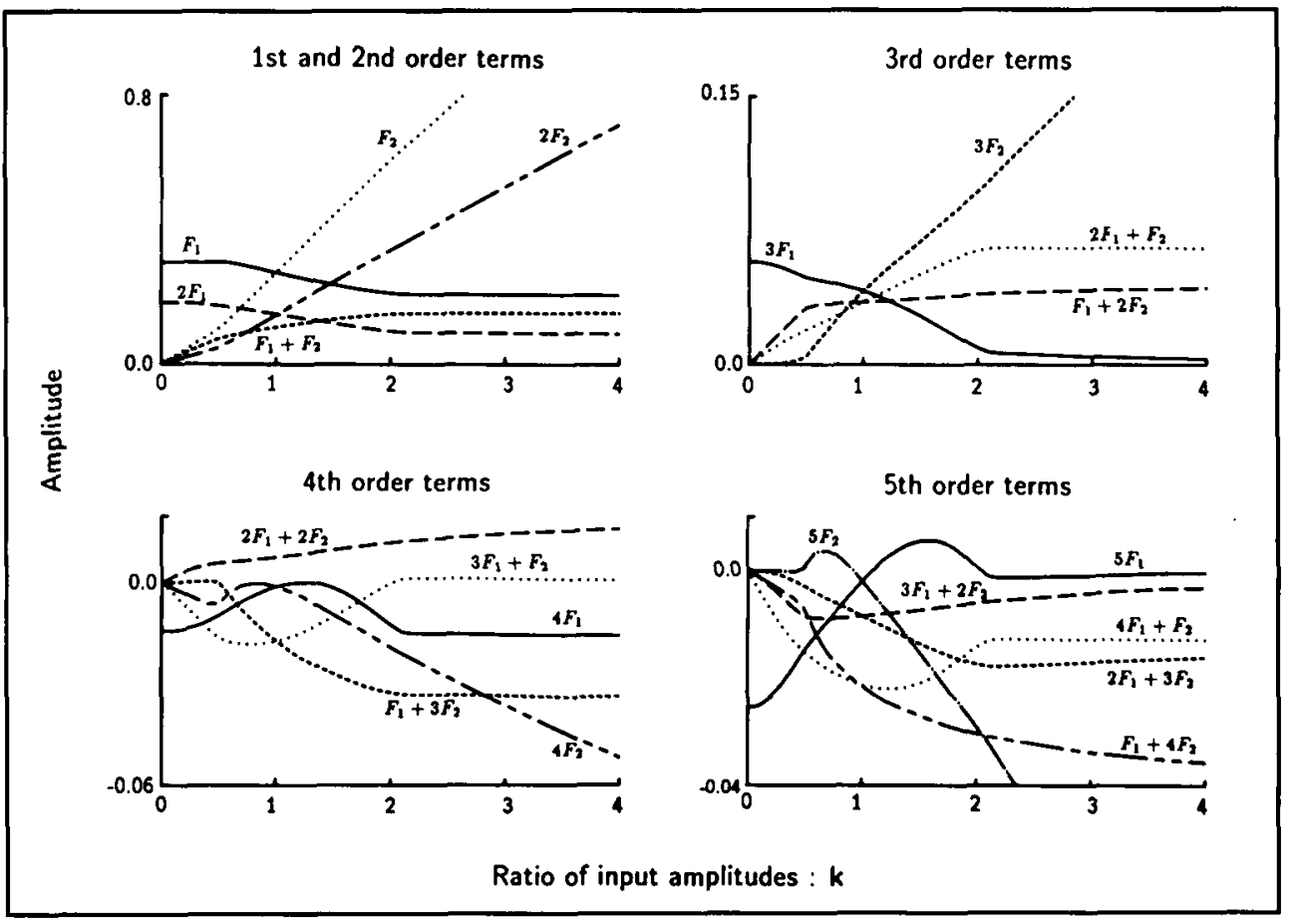

B

Figure 6 - Theoretical results. $6 A$ - Ordinates plot the amplitudes of some of the various frequency components in the output of a square root halfwave rectifier fed by two sinewaves of frequency $F_{1} \mathrm{~Hz}$ and $F_{2} \mathrm{~Hz}$. The ratio between the $F_{2}$ input's amplitude and the $F_{1}$ input's amplitude is plotted along the abscissa. $6 B$-As in A, but for two linear half-wave rectifiers in parallel converging onto a third rectifier (compressive, squareroot).

AC-coupled; (2) two compressive (squareroot) rectifiers in series, AC-coupled; (3) a rectifier whose shape resembles the proposed contrast transducer function of the human eye, 23 namely a threshold followed by an initial accelerating segment followed by a compressive segment; (4) an accelerating (square law) rectifier followed by a compressive (thirdroot) rectifier, AC-coupled. Figure 7A shows that the second order curves are fairly similar, but when the third order terms are taken into 


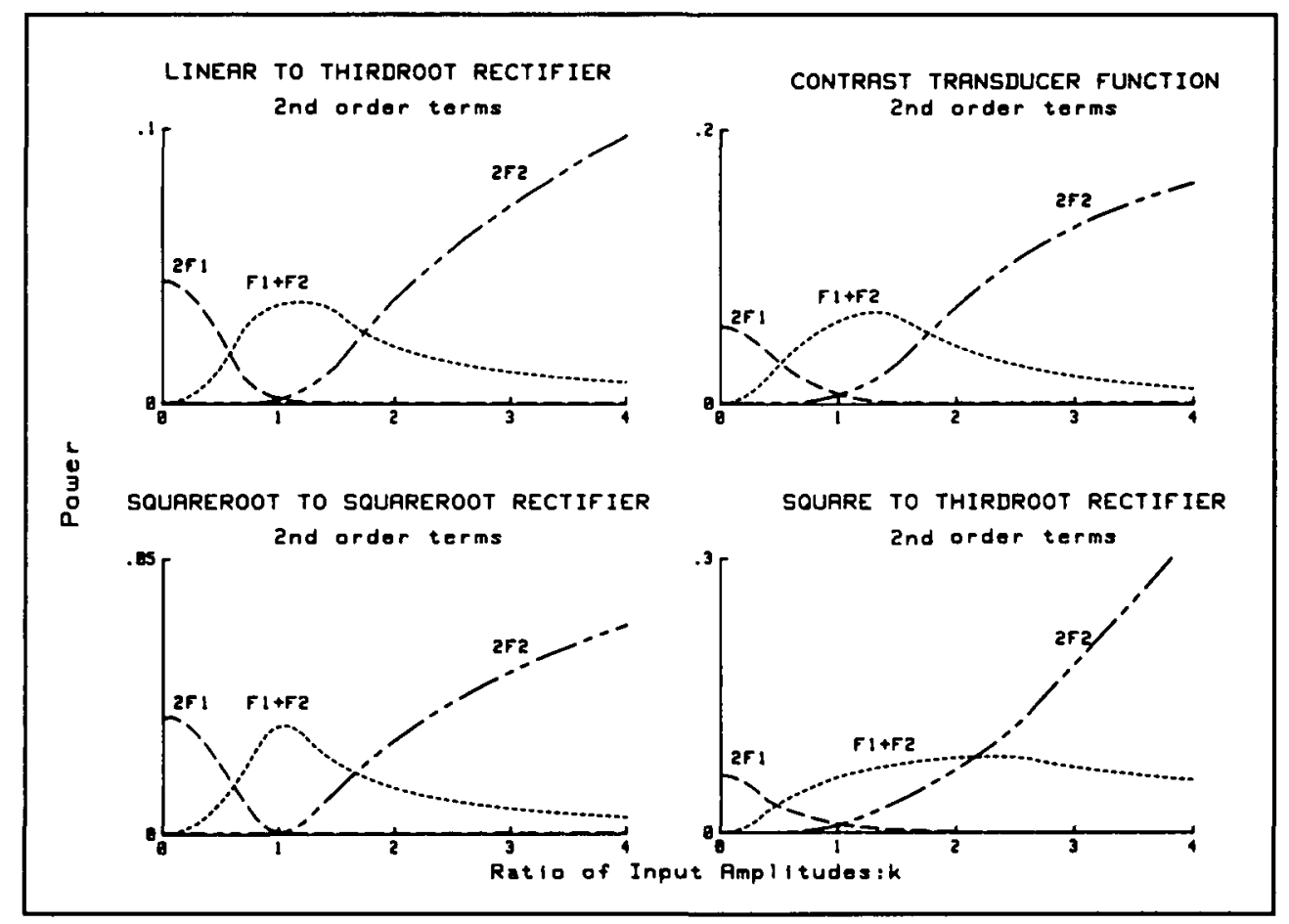

A

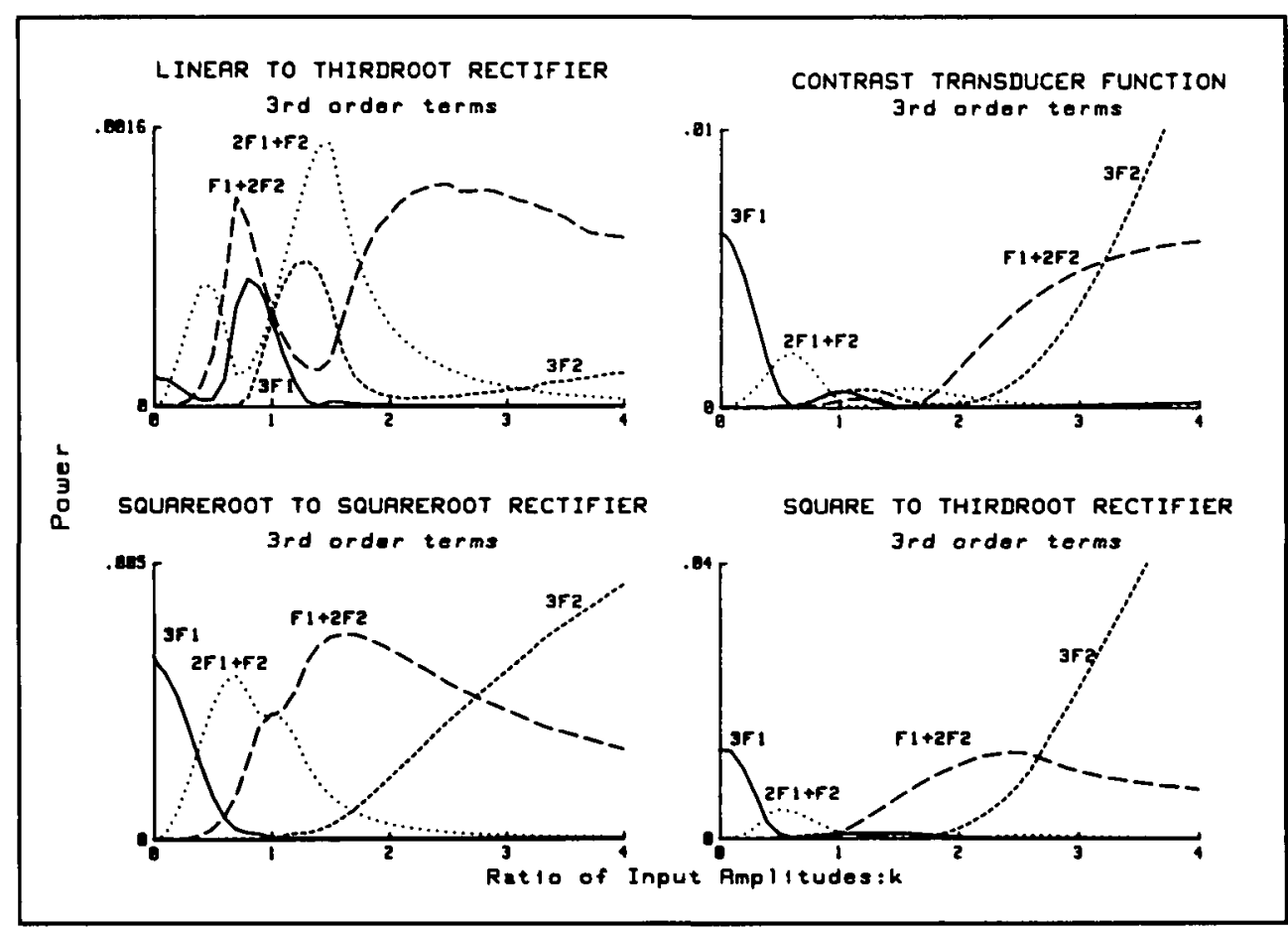

B

Figure 7A-Second order terms for four different rectifier models. The curves are not strikingly different.

Figure $7 B-$ Third order terms for the same four rectifier models. The curves are quite different.

account (Figure 7B) the families of curves are easily distinguished.

Finally, we should briefly consider the effects of linear frequency-filters. A linear frequency filter placed before the rectifying stage in Figure 6A would alter the effective value of $k$ depending on the absolute values of $F_{1}$ and $F_{2}$ relative to the filter's tuning curve, thus shifting the curves bodily along the abscissa. This shift can be rendered indefinitely small by minimising the difference between $F_{1}$ and $F_{2}$. Again, a linear filter placed after the rectifying stage would bodily shift the curves 
along the ordinate without affecting their shapes, the amount of shift depending on the absolute frequency of the component in relation to the filter's tuning curve. ${ }^{11,15}$ For simplicity we will not further discuss the effect of linear filters. In the experiments described below $\left(F_{1}-F_{2}\right)$ was small, and we emphasize the shapes of the curves rather than their absolute amplitudes.

\section{Experimental}

Figure 8 shows a short section of the EEG spectrum analyzed at a resolution of $0.0039 \mathrm{~Hz}$. This dichoptic record shows one cross-modulation term (boxed) between the $2 F_{1}$ and $2 F_{2}$ second harmonic components. The dichoptic $\left(F_{1}+F_{2}\right) \mathrm{Hz}$ component reflects a nonlinear interaction between the signals from left and right eyes. Clearly, this term could not arise before the convergence of the signals from the right and left eyes. $\dagger$

Figure 9 plots powers of EP response components versus the modulation depth of the $F_{2} \mathrm{~Hz}$ flicker stimulus (from $0 \%$ to $70 \%$ ). The procedure for modelling the responsible nonlinearities is to compare families of curves such as those shown in Figure 9 with corresponding families of curves that have been theoretically calculated for candidate models.

The models considered for the Figure 9 dichoptic data comprised: (1) Two linear monocular channels converging onto a binocular rectifier, (2) the linear sum of two identical monocular channels, each containing a rectifier and (3) two identical monocular channels each containing a rectifier, converging onto a binocular rectifier. The families of curves for models 1,2 and 3 were calulated for the cases of linear, compressive and accelerating half-wave rectifiers. Model 1 could be rejected when the $3 F_{2}$ term was taken into account as well as second order terms. Model 2 could be rejected because it does not provide any of the cross modulation terms evident in the data of Figure 8.
Model 3 provided the best fit to the data of the parallel/cascaded models. The data were better fit when two monocular linear rectifiers fed a binocular compressive (square root) rectifier than when all three rectifiers were identical linear, compressive (square or third root) or accelerating ( 2 nd power) rectifiers.

\section{Discussion}

This paper describes an extension of the two-sinewave EP method that allows multi-neuron models to be tested experimentally by comparing EP data with a library of theoretically-

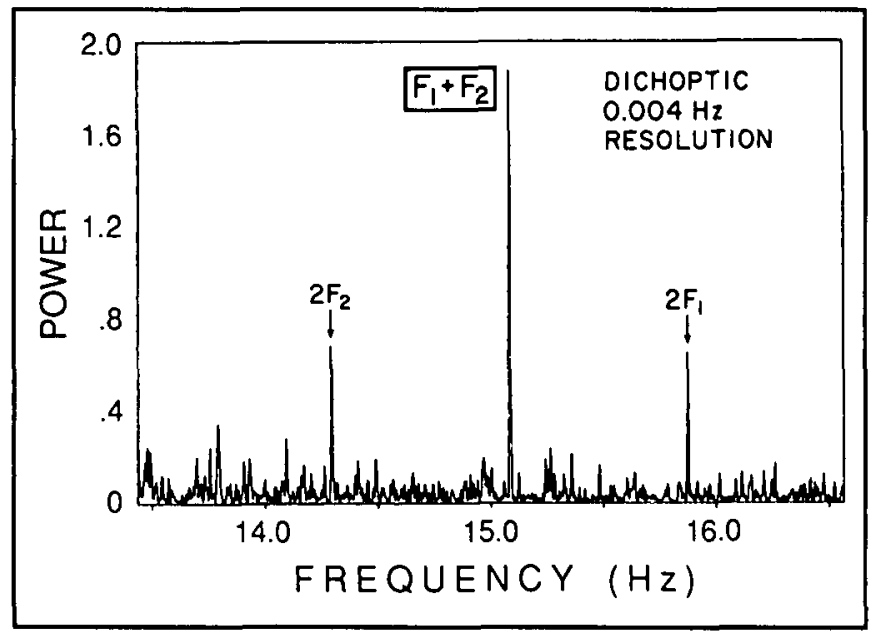

Figure 8 - One eye viewed homogeneous field flicker of $F_{1}=8 \mathrm{~Hz}$ while the other eye viewed a similar homogeneous field flickering at $F_{2}=7 \mathrm{~Hz}$. The EEG spectrum was recorded at a resolution of $0.004 \mathrm{~Hz}$ by zoom-FFT. The $\left(F_{1}+F_{2}\right)$ EP component is due to nonlinear interaction between signals from left and right eyes.

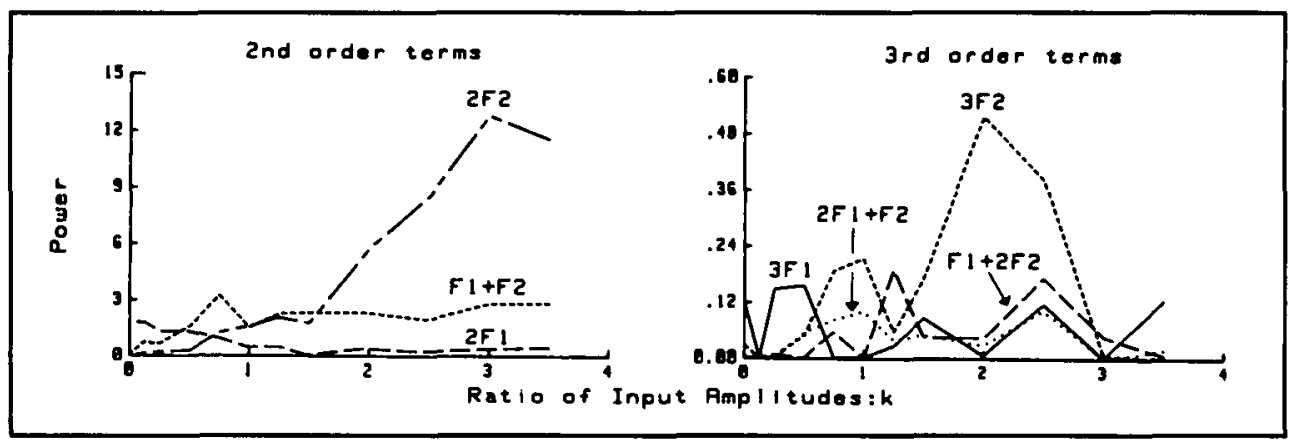

Figure 9 - Evoked potential data. Ordinates plot the amplitudes of some of the various frequency components in the evoked potential recorded while a subject viewed a homogeneous field flickering at $8 \mathrm{~Hz}$ with $20 \%$ modulation depth in the left eye while a second homogeneous field flickering at $7 \mathrm{~Hz}$ viewed by the right eye was varied in modulation depth from $0 \%$ to $70 \%$.

†A cross-modulation component to pattern stimulation has been reported when the left eye views a pattern of 20 min arc checks reversing $F_{1}$ times per second while the right eye views a pattern of $20 \mathrm{~min}$ arc checks reversing $F_{2}$ times per second, the two patterns being binocularly-fused. 24.25 Surprisingly, the dichoptic component described had a frequency of $1 / 2\left(F_{1}+F_{2}\right)$ rather than $\left(F_{1}+F_{2}\right)$. If the binocular interaction occurred after generation of the $F_{1} \mathrm{~Hz}$ and $F_{2} \mathrm{~Hz}$ pattern-reversal responses we would expect the lowest frequency in the cross-modulation term to be $F_{1} \mathrm{~Hz}$ or $F_{2} \mathrm{~Hz}$. If the stimuli were indeed quite free from artifactual $F_{1} \mathrm{~Hz}$ and $F_{2} \mathrm{~Hz}$ overall luminance flicker, this finding suggests that the $1 / 2\left(F_{1}+F_{2}\right)$ term was generated by a binocular interaction between signals generated by local luminance changes. This would require that local retinal responses to local luminance flicker wère kept segregated right up to the stage of binocular interaction and that the receptive field size of these segregated parallel 'lines' was no larger than the stimulus check size (i.e. 20 min arc). 
obtained predictions for candidate models. In order to use this approach we have developed an ultra-high resolution method for wide-bandwidth EP recording and a theoretical procedure for obtaining the predictions of multi-neuron models.

In brief, the experimental procedure is to simultaneously stimulate with two frequencies $F_{1}$ and $F_{2}$, holding the amplitude of one input constant while varying the amplitude of the second input. This gives a family of plots of EP amplitude versus stimulus amplitude, one plot for each of the many $\left(n F_{1} \pm m F_{2}\right)$ frequency components in the EEG. As more and more of the frequency components are taken into account, the corresponding family of plots for any given neural model becomes a more and more specific 'fingerprint' of that model, thus allowing confident rejection of candidate models. Compared with the wellknown Wiener kernel (white-noise) time-domain method of EP analysis, ${ }^{26}$ this frequency-domain approach has the advantage that many of the high-order response components (e.g. $n+m=$ 3 to 6 ) can readily be measured physiologically thus, as just mentioned, allowing sharp discrimination between candidate models. This advantage is empirical and stems from the very narrow bandwidths (e.g. $<0.004 \mathrm{~Hz}$ ) of the EP components. Because noise is continuousiy distributed along the frequency axis, comparatively little falls into any given $0.004 \mathrm{~Hz}$ frequency bin. But any single bin may contain almost the entire power of an EP component, thus giving high signal-to-noise ratios. In the time domain, however, signal substantially overlaps with noise, and Wiener kernel analysis is a time domain procedure. Our approach can be used in audition 27 as well as in vision, 7,16 and is also convenient for investigating cross-modality interactions. Note that our approach is not a general method. For example, it does not apply to systems whose properties change with time or whose properties depend on the previous history of stimulation. (These limitations also apply to the Wiener kernel method).

In applying our approach to binocular interaction we should note that methods for recording EPs to disparity change, $28,29,30$ EPs to correlation/anticorrelation 29,31,32 and interocular suppression of pattern $\mathrm{EPs}^{33}$ all fail when visual acuity is low in one or in both eyes, so that these techniques are of limited effectiveness in assessing binocular interaction in amblyopic children or infants. On the other hand, recording cross-modulation terms produced by dichoptic stimulation with unpatterned-field flicker provide a means of measuring binocularity that does not require good visual acuity or accurate binocular convergence. Above we compare binocular and dichoptic EP data with theoretical predictions so as to distinguish between nonlinearities sited before and after binocular convergence. Finally we note that, by using LED stimulators mounted in goggles, the technique might, perhaps, be used to monitor binocularity in young children undergoing therapy for amblyopia.

\section{ACKNOWLEDGEMENTS}

This research was supported by the Medical Research Council of Canada and sponsored by the U.S. Air Force Office of Scientific Research. Artwork by Paragon Services.

\section{REFERENCES}

1. Regan D. Evoked Potentials in Psychology, Sensory Physiology and Clinical Medicine. London: Chapman and Hall; New York; Wiley 1972.

2. Regan D. Human Brain Electrophysiology: Evoked Potentials and Evoked Magnetic Fields in Science and Medicine, New York: Elsevier, 1989.

3. Dawson GD. A summation technique for the detection of small evoked potentials. Electroenceph Clin Neurophysiol 1954; 6: 65-84.

4. Halliday AM. (ed.). Evoked Potentials in Clinical Testing. Churchill Livingstone, London 1982.

5. Chiappa KH. Evoked Potentials in Clinical Medicine. Raven, New York 1983.

6. Regan D. Some characteristics of average steady-state and transient responses evoked by modulated light. Electroenceph $\mathrm{Clin}$ Neurophysiol 1966; 20: 238-248.

7. Regan D, Regan MP. Nonlinearity in human visual responses to two-dimensional patterns, and a limitation of Fourier methods. Vision Res. 1987; 27: 2181-2183.

8. Fiorentini A, Pirchio M, Spinelli D. Electrophysiological evidence for spatial frequency selective mechanisms in adults and infants. Vision Res 1983; 23: 119-127.

9. Regan D. Spatial frequency mechanisms in human vision investigated by evoked potential recording. Vision Res 1983; 23: 14011408.

10. Zemon V, Ratliffe F. Intermodulation components of the visual evoked potential: Responses to lateral and superimposed stimuli. Bio Cybernet 1984; 50: 401-408.

11. Spekreijse H. Analysis of EEG Responses in Man (Thesis) DW Junk, The Hague 1966.

12. Schiller PH. The central visual system. Vision Res. 1986; 26: 13511386.

13. Barlow HB, Levick WR. The mechanism of directionally selective units in the rabbit's retina. J Physiol 1965; 178: 477-504.

14. Spekreijse H, Oosting H. Linearising: A method for analysing and synthesising nonlinear systems. Kybernetik 1970; 7: 22-31.

15. Spekreijse H, Reits D. Sequential analysis of the visual evoked potential system in man: nonlinear analysis of a sandwich system. Ann NY Acad Sci 1982; 388: 72-97.

16. Regan MP, Regan D. Monocular and binocular nonlinearities in flicker evoked potentials. Spekreijse and van der Tweel's linearization revisited after 21 years. Third Internat Evoked Potential 1986; Symp., Berlin.

17. Regan MP, Regan D. A frequency domain technique for characterizing nonlinearities in biological systems. J. Theor. Biol 1988; 133: 293-317.

18. Bennett WR. New results in the calculation of modulation products. Bell System Tech J 1933; 228-243.

19. Gabor D. Theory of communication. J Inst Elect Eng (Lond) 1946; 93: 429-456.

20. Thrane N. Zoom-FFT. Bruel and Kjaer Tech Rev 1980; 1980; 2: 341.

21. Regan D. Rapid objective refraction using evoked brain potentials. Invest Ophthalmol 1973; 12: 669-79.

22. Regan D. Electrophysiological evidence for colour channels in human pattern vision. Nature 1974; 250: 437-449.

23. Wilson HR. A transducer function for threshold and suprathreshold human vision. Biol Cybernetics 1980; 38: 171-178.

24. Katsumi O, Oguchi Y, Kawara T, et al. Effects of stimulus contrast on binocular VEP. Jap J Ophthalmol 1984; 28: 401-408.

25. Uemura Y, Katsumi O. A new approach to the objective evaluation of binocular function. In: Reinecke RD (ed.) Strabismus II, Grune and Stratton, New York 1984; 511-521.

26. Marmarelis PZ, Marmarelis VZ. Analysis of Physiological Systems. The White Noise Approach. Plenum Press, New York 1978.

27. Regan D, Regan MP. The transducer characteristic of hair cells in the human inner ear: a possible objective measure. Brain Res 1988; 438: 363-365. 
28. Regan D, Spekreijse H. Electrophysiological correlate of binocular depth perception in man. Nature 1970; 255: 92-94.

29. Julesz B, Kropf1 W, Petrig B. Large evoked potentials to dynamic random dot correlograms and stereograms permit quick determination of stereopsis. Proc Natl Acad Sci USA 1980; 77: 23482351.

30. Norcia AM, Sutter EE, Tyler CW. Electrophysiological evidence for the existence of coarse and fine disparity mechanisms in human. Vision Res 1985; 25: 1603-1611.

31. Herpers MJ, Caberg HB, Mol JMF. Human cerebral potentials evoked by moving dynamic random dot stereograms. Electroenceph Clin Neurophysiol 1981; 52: 50-56.
32. Lehmann D, Skrandies W, Lindenmaier C. Sustained cortical potentials evoked in humans by binocularly correlated, uncorrelated and disparate dynamic random-dot stimuli. Neurosci Lett 1978; 10: 129-134.

33. Spekreijse H, van der Tweel LH, Regan D. Interocular sustained suppression: correlations with evoked potential amplitude and distribution. Vision Res 1972; 12: 521-526. 